\title{
Supplementation of Enzymatic and Non-enzymatic Antioxidants to the Extender improves Sperm Functionality during Storage at $4^{\circ} \mathrm{C}$ in Labrador Dog
}

\author{
Ranjna S Cheema* and Sukhjeet Kaur \\ Department of Veterinary Gynecology and Obstetrics, Guru Angad Dev Veterinary and Animal Sciences University, \\ Ludhiana, Punjab, INDIA \\ "Corresponding author: RS Cheema; E-mail: ranjna.cheema@gmail.com
}

Received: 02 May, 2020

Revised: 28 Jan., 2021

Accepted: 09 Feb., 2021

\begin{abstract}
Several extenders to preserve fertilizing capacity of preserved canine semen have been successfully tested, but further studies are requisite to improve its quality. Effect of supplementation of superoxide dismutase (SOD), catalase (CAT), glutathione peroxidase (GPX) and glutathione (GSH) to Tris-citric acid-fructose-egg yolk extender on Labrador dog sperm survival during storage at $4^{\circ}$ was evaluated. Different concentrations per antioxidant i.e. SOD (50-300 IU/ml), GPX (1.5-2.5 IU/ml), CAT (100$400 \mu \mathrm{g} / \mathrm{ml})$ and GSH $(2.5-10 \mu \mathrm{M} / \mathrm{ml})$ were evaluated to look for an optimum dose. Semen was analyzed for motility, viability and plasma membrane integrity (PMI) after every $24 \mathrm{hrs}$ till $72 \mathrm{hrs}$ of preservation. Semen was also analyzed for acrosome integrity (AI) at $0,72 \mathrm{hrs}$ and lipid peroxidation at $72 \mathrm{hrs}$ of storage. Values for motility, viability, PMI, AI were significantly $(\mathrm{p}<0.05)$ higher in the presence of 200IU/ $\mathrm{ml} \mathrm{SOD}, 2 \mathrm{IU} / \mathrm{ml}$ GPX, $200 \mu \mathrm{g} / \mathrm{ml}$ CAT and $7.5 \mu \mathrm{M} / \mathrm{ml}$ GSH compared to control and other tested doses. MDA concentration was non-significantly ( $p>0.05$ ) less in the presence of all doses of SOD and 2.0 IU GPX compared to control at $72 \mathrm{hrs}$ of preservation. MDA concentration in the presence of $200 \mu \mathrm{g} / \mathrm{ml}$ catalase and $7.5 \mu \mathrm{M} / \mathrm{ml} \mathrm{GSH}$ was non-significantly $(\mathrm{p}>0.05)$ higher than control. It was concluded that SOD, catalase, GPX and GSH at a concentration of $200 \mathrm{IU}, 2.0 \mathrm{IU}, 200 \mu \mathrm{g}$ and $7.5 \mu \mathrm{M}$ per ml were optimum concentrations to be supplemented to the extender for positive effect. Supplementation of antioxidants could improve sperm attributes by maintaining proportionate level of oxidative stress during preservation of Labrador dog semen at $4^{\circ}$ for $72 \mathrm{hrs}$.
\end{abstract}

\section{HIGHLIGHTS}

( Effect of supplementation of different doses of antioxidants was observed on semen during preservation at $4{ }^{\circ} \mathrm{C}$.

0 SOD, catalase, GPX and GSH at a concentration of $200 \mathrm{IU}, 2.0 \mathrm{IU}, 200 \mu \mathrm{g}$ and $7.5 \mu \mathrm{M}$ per ml were optimum concentrations to be supplemented to the extender for positive effect

Keywords: Antioxidants, preservation, TEY extender, $4^{\circ} \mathrm{C}$, Labrador dog semen

Artificial insemination (AI) with fresh semen in canines presents pregnancy rates almost similar to natural mating. The use of chilled or cryopreserved semen leads to the reduction in fertility rate (Yu et al., 2002). It was verified that $\mathrm{AI}$ done with cryopreserved semen in dogs results in $30 \%$ reduced pregnancy rate and $31 \%$ lower litter size, compared to inseminations with fresh semen (LindeForsberg and Forsberg, 1993). The metabolic activities of spermatozoa during storage need to be reduced by using an appropriate medium and storage temperature. Several extenders to preserve fertilizing capacity of canine semen have been successfully tested, but further studies are requisite to improve the quality of preserved semen (Kasimanickam et al., 2012). There is little available information about the molecular mechanisms leading to death of spermatozoa during cooling, and apoptosis may be the reason (Tapia et al., 2012). Sperm survival during

How to cite this article: Cheema, R.S. and Kaur, S. (2021) Supplementation of enzymatic and non-enzymatic antioxidants to the extender improves sperm functionality during storage at $4^{\circ} \mathrm{C}$ in labrador dog. J. Anim. Res., 11(1): 71-79.

Source of Support: None; Conflict of Interest: None 
storage at $4^{\circ} \mathrm{C}$ depends on oxygen consumption and metabolism, which increase the amount of reactive oxygen species (Agarwal et al., 2003). Low ROS amounts are required for fertilizing capabilities, as they are involved in kinetic function, sperm capacitation, hyperactivation and fusion of the oocyte with the spermatozoa (Rota et al., 1995). Excessive ROS production can damage sperm function, sperm- oocyte fusion, and impair fertilization.

ROS concentration is controlled by antioxidants of the seminal fluid and inside the sperm cell, thereby avoiding ROS overload and preserving semen quality (Michael et al., 2003; Tapia et al., 2012). Antioxidant systems consist of enzymatic antioxidants, such as superoxide dismutase (SOD), catalase (CAT), glutathione peroxidase (GPx) and non-enzymatic antioxidants [glutathione (GSH), amino acids, vitamin A, C, E, and uric acid. A study on dogs confirmed the presence of endogenous antioxidants in the seminal plasma of pre-spermatic, spermatic, and post-spermatic fractions, with SOD representing the major enzymatic antioxidant in all dog ejaculate fractions, whereas GPX activity was present in the sperm-rich and post-spermatic fractions, and CAT activity was deficient (Strzeżek et al., 2009). CAT activity in dog ejaculates is demonstrated and the addition of SOD and CAT in the dilution extender of canine semen improved sperm quality (Kawakami et al., 2007). In canine semen cryopreservation, seminal plasma is removed by centrifugation (Sirivaidyapong et al., 2001) and hence there is elimination of natural antioxidants (Strzeżek et al., 2009) and the sparse cytoplasmic volume in the sperm cell mid-piece limits its antioxidant capacity (Aitken et al., 1996). Antioxidants supplementation to the extender allows the improvement of sperm quality after thawing and higher sperm longevity, which is considered an important indicator to the successful cryopreservation in dogs (Monteiro et al., 2009). Lucio et al., 2016) observed a higher acrosome protection and higher pregnant bitches with sperm cryopreserved with $10 \mathrm{mM}$ of reduced glutathione (GSH). Hence, the aim of this study was to investigate the effect of supplementation of SOD, CAT, GPX and GSH to Tris-citric acid-fructose-egg yolk extender on Labrador dog sperm survival during storage at $4^{\circ} \mathrm{C}$.

\section{MATERIALS AND METHODS}

\section{Experimental design}

Antioxidants were purchased from Sigma-Aldrich Company. Study was conducted in two phases. Effect of supplementation of SOD, GPx, CAT and GSH to TEY buffer was observed on Labrador dog semen stored at $4^{\circ} \mathrm{C}$ from 0-72 hrs. Different concentrations per antioxidant i.e. SOD (50-300 IU/ml), GPx (1.5-2.5 IU/ml), CAT (100-400 $\mu \mathrm{g} / \mathrm{ml})$ and GSH $(2.5-10 \mu \mathrm{M} / \mathrm{ml})$ were evaluated to look for an optimum dose.

\section{Maintenance of dogs and semen collection}

All the procedures were approved by the CPCSEA, New Delhi vide F. No 25-19-2018-CPCSEA, dated 22/11/2018. Six Labrador dogs were maintained in individual pens. They were daily fed $500 \mathrm{~g}$ cooked feed twice daily and water provided ad libitum. Dogs were given regular exercise in the form of walking / running for one hour daily in the morning and evening. Dogs were dewormed and vaccinated for rabies, CDV, CAV2, CPV, CPI and CAV1. Semen was collected by message method twice a week.

\section{Preparation of extenders for extension of semen}

Tris - citric acid-fructose buffer (Tris, $3.08 \mathrm{~g}$; citric acid, $1.78 \mathrm{~g}$; fructose, $1.25 \mathrm{~g} / 100 \mathrm{ml}, \mathrm{pH} 7.2$, gentamycin, $5 \mathrm{mg}$ ) was prepared and autoclaved at $15 \mathrm{lb}$ for $15 \mathrm{~min}$. Buffer was filtered through 0.2-micron membrane filter. Egg yolk and gentamycin at a concentration of $15 \%$ and $50 \mu \mathrm{g} / \mathrm{ml}$ were added to the buffer on the day of experiment, again filtered through 0.2-micron membrane filter and kept at $37^{\circ} \mathrm{C}$ (TEY extender).

\section{Processing of semen for storage at $4^{\circ} \mathrm{C}$}

Semen of dogs exhibiting $>70 \%$ motility was pooled for each trial and divided into required aliquots. To each aliquot SOD $(50,100,200$ and $300 \mathrm{IU} / \mathrm{ml}), \operatorname{GPX}(1.5,2.0$, $2.5 \mu \mathrm{M} / \mathrm{ml})$, CAT $(100,200,300,400 \mathrm{IU} / \mathrm{ml})$ and GSH $(2.5,5.0,7.5$ and $10 \mu \mathrm{M} / \mathrm{ml})$ were added and incubated at $37^{\circ} \mathrm{C}$ for $10 \mathrm{~min}$. To each aliquot, extender was added and semen-extender mixture was centrifuged at $960 \mathrm{~g}$ for 3 
min. Loose pellet was re-suspended in extender containing respective concentration of the antioxidant to get a final sperm concentration between 150-200 spermatozoa / ml. Sperm suspension was again equilibrated at $37^{\circ} \mathrm{C}$ for 10 min and evaluated for motility, viability, membrane and acrosome integrity. Samples in tubes were shifted to a container containing warm water $\left(37^{\circ} \mathrm{C}\right)$ and placed in a cooling cabinet at $4^{\circ} \mathrm{C}$. Semen was analyzed for motility, viability and membrane integrity after every $24 \mathrm{hrs}$ till $72 \mathrm{hrs}$ of storage. Semen was also analyzed for acrosome integrity at $0,72 \mathrm{hrs}$ and lipid peroxidation at $72 \mathrm{hrs}$ of storage.

\section{Evaluation of semen}

Motility was evaluated by wet mount and track method. Viability was assessed by staining the spermatozoa with syber green-propidium iodide stain kit (Sigma). Hypoosmotic swelling test (HOST) was performed to analyze the integrity of sperm membrane (Jeyendran et al., 1984). One drop of semen incubated in 60 mosm HOS solution for $30 \mathrm{~min}$ was placed on a slide, covered with cover slip and examined under bright field microscope (Olympus) at $400 \mathrm{X}$ for coiled tailed spermatozoa. A control was also run in PBS, pH 7.4. The number of coiled tailed spermatozoa in PBS was deducted from the number in hypo-osmotic solution and the resultant figure was taken as the HOS-reactive spermatozoa. For acrosome integrity, sperm smears prepared on clean glass slides were stained with spermac stain kit (Minitube) as per manufacturer's instructions. About 200 spermatozoa were counted in different fields for all sperm attributes and percentage motility, viability, plasma membrane integrity and acrosome integrity were calculated.

Malondialdehyde concentration (end product of LPO) was estimated by the method of Buege et al., 1978). Briefly, semen was washed twice with PBS, $\mathrm{pH} 7.4$ by centrifugation at $865 \mathrm{~g}$ for $5 \mathrm{~min}$. Sperm pellet was suspended in $0.2 \mathrm{ml} \mathrm{PBS}$, mixed with $0.1 \mathrm{ml}$ of $150 \mathrm{mM}$ Tris $\mathrm{HCl}(\mathrm{pH} 7.1)$ and incubated at $37^{\circ} \mathrm{C}$ for $20 \mathrm{~min}$. After incubation, $0.5 \mathrm{ml} 10 \%$ TCA and $1.0 \mathrm{ml} 0.375 \%$ TBA were added and kept for $20 \mathrm{~min}$ in the boiling water bath. Thereafter, mixture was centrifuged for $15 \mathrm{~min}$ at $2500 \mathrm{~g}$ and absorbance of supernatant was taken at $532 \mathrm{~nm}$. The molar extinction coefficient for MDA was calculated as below:
MDA content $($ units $)=$

$$
\text { OD } \times \text { volume of assay mixture }
$$

Volume of sample taken $\times$ coefficient extinction

\section{Statistical Analysis}

Significant differences (5\% level) among the extenders were tested by one-way Anova using SPSS 16 program (Student version for windows, SPSS Inc. 233 South Wacker Drive, $11^{\text {th }}$ floor Chicago, IL 60606-6412). Normality of the data was assessed using the ShapiroWilk test and homogeneity of variances was evaluated using the Levene test.

\section{RESULTS AND DISCUSSION}

\section{Effect of antioxidants on freshly extended semen}

Treatment of fresh semen with different concentrations of SOD and GPX at $37^{\circ}$ for $10 \mathrm{~min}$ did not show significant difference in sperm attributes of freshly extended semen. However, treatment with $200 \mu \mathrm{g} / \mathrm{ml}$ catalase and $7.5 \mu \mathrm{M} /$ $\mathrm{ml}$ GSH exhibited significantly higher values for sperm attributes in freshly extended semen compared to control and other tested doses (Table 1).

\section{Effect of Superoxide dismutase on storage of semen at $4^{\circ} \mathrm{C}$}

Preservation of semen in TEY extender supplemented with 100 and 200 IU SOD revealed significantly higher values of individual motility, viability and PMI at $24 \mathrm{hrs}, 48 \mathrm{hrs}$ and $72 \mathrm{hrs}$ compared to control and $300 \mathrm{IU}$ (Table 1). Values for motility, viability and PMI were non-significantly $(p>0.05)$ higher in extender supplemented with 200 IU SOD than $100 \mathrm{IU}$ during storage of semen at $4^{\circ} \mathrm{C}$ from $24-72 \mathrm{hrs}$. Acrosome integrity was non-significantly different among the freshly extended - control and SOD treated semen. But it was significantly $(\mathrm{p}<0.05)$ higher in the presence of 200 IU SOD than control and other concentrations at $72 \mathrm{hrs}$ of preservation. MDA production ( $\mu \mathrm{M} / 10^{9}$ spermatozoa) was non-significantly $(\mathrm{p}>0.05)$ higher in control than SOD treated semen samples at $72 \mathrm{hrs}$ of preservation. 
Table 1: Effect of supplementation of superoxide dismutase (100-300 IU/ml) to TEY extender on sperm attributes during storage of Labrador dog semen at $4^{\circ} \mathrm{C}$ for $72 \mathrm{hrs}$

\begin{tabular}{|c|c|c|c|c|c|c|c|c|c|c|c|c|c|c|c|c|}
\hline \multirow{2}{*}{$\begin{array}{l}\text { Sperm } \\
\text { parameters }\end{array}$} & \multicolumn{4}{|c|}{$\mathbf{0} \mathbf{H r}^{\mathbf{A}}$} & \multicolumn{4}{|c|}{$24 \mathrm{Hrs}^{\text {B }}$} & \multicolumn{4}{|c|}{$48 \mathrm{Hrs}^{\mathrm{C}}$} & \multicolumn{4}{|c|}{$72 \mathrm{Hrs}^{\mathrm{D}}$} \\
\hline & 0 & 100 & 200 & 300 & 0 & 100 & 200 & 300 & 0 & 100 & 200 & 300 & 0 & 100 & 200 & 300 \\
\hline MOT (\%) & $\begin{array}{l}80.0^{\mathrm{b}} \\
\pm 0.0\end{array}$ & $\begin{array}{l}86.7^{\mathrm{ab}} \\
\pm 1.7\end{array}$ & $\begin{array}{l}85.5^{\mathrm{a}} \\
\pm 2.9\end{array}$ & $\begin{array}{l}80.4^{\mathrm{b}} \\
\pm 2.9\end{array}$ & $\begin{array}{l}68.3^{\mathrm{b}} \\
\pm 1.7\end{array}$ & $\begin{array}{l}75.0^{\mathrm{b}} \\
\pm 0.0\end{array}$ & $\begin{array}{l}78.7^{\mathrm{a}} \\
\pm 1.3\end{array}$ & $\begin{array}{l}71.7^{\mathrm{b}} \pm \\
3.3\end{array}$ & $\begin{array}{l}63.3^{\mathrm{b}} \\
\pm 4.4\end{array}$ & $\begin{array}{l}68.3^{\mathrm{b}} \\
\pm 1.7\end{array}$ & $\begin{array}{l}71.7^{\mathrm{a}} \\
\pm 3.3\end{array}$ & $\begin{array}{l}65.5^{\mathrm{b}} \\
\pm 5.0\end{array}$ & $\begin{array}{l}56.7^{\mathrm{b}} \\
\pm 3.3\end{array}$ & $\begin{array}{l}56.7^{\mathrm{b}} \pm \\
4.4^{4}\end{array}$ & $\begin{array}{l}=61.7^{\mathrm{a}} \\
\pm 6.0\end{array}$ & $\begin{array}{l}56.7^{\mathrm{b}} \pm \\
6.0\end{array}$ \\
\hline VIA (\%) & $\begin{array}{l}86.0^{\mathrm{ab}} \\
\pm 0.9\end{array}$ & $\begin{array}{l}92.2^{\mathrm{a}} \pm \\
1.3\end{array}$ & $\begin{array}{l}90.0^{\mathrm{ab}} \\
\pm 5.5\end{array}$ & $\begin{array}{l}86.5^{\mathrm{b}} \\
\pm 4.8\end{array}$ & $\begin{array}{l}74.0^{\mathrm{b}} \\
\pm 2.9\end{array}$ & $\begin{array}{l}81.6^{\mathrm{a}} \\
\pm 0.5\end{array}$ & $\begin{array}{l}83.9^{\mathrm{a}} \\
\pm 6.2\end{array}$ & $\begin{array}{l}77.0^{\mathrm{ab}} \\
\pm 3.3\end{array}$ & $\begin{array}{l}69.2^{\mathrm{b}} \\
\pm 4.0\end{array}$ & $\begin{array}{l}75.4^{\mathrm{a}} \\
\pm 1.3\end{array}$ & $\begin{array}{l}77.5^{\mathrm{a}} \\
\pm 3.9\end{array}$ & $\begin{array}{l}71.6^{\mathrm{b}} \\
\pm 5.2\end{array}$ & $\begin{array}{l}63.2^{\mathrm{b}} \\
\pm 2.2\end{array}$ & $\begin{array}{l}66.9^{\mathrm{ab}} \\
\pm 2.2\end{array}$ & $\begin{array}{l}71.8^{\mathrm{a}} \\
\pm 3.3\end{array}$ & $\begin{array}{l}64.9^{\mathrm{ab}} \\
\pm 1.9\end{array}$ \\
\hline PMI (\%) & $\begin{array}{l}75.8^{\mathrm{b}} \\
\pm 1.5\end{array}$ & $\begin{array}{l}82.0^{\mathrm{a}} \pm \\
1.5\end{array}$ & $\begin{array}{l}82.4^{\mathrm{a}} \\
\pm 3.3\end{array}$ & $\begin{array}{l}79.7^{\mathrm{a}} \\
\pm 3.0\end{array}$ & $\begin{array}{l}70.0^{\mathrm{b}} \\
\pm 2.2\end{array}$ & $\begin{array}{l}76.1^{\mathrm{ab}} \\
\pm 2.2\end{array}$ & $\begin{array}{l}79.2^{\mathrm{a}} \\
\pm 1.5\end{array}$ & $\begin{array}{l}73.1^{\mathrm{ab}} \\
\pm 2.8\end{array}$ & $\begin{array}{l}63.5^{\mathrm{b}} \\
\pm 3.1\end{array}$ & $\begin{array}{l}71.3^{\mathrm{a}} \\
\pm 1.3\end{array}$ & $\begin{array}{l}74.1^{\mathrm{a}} \\
\pm 3.8\end{array}$ & $\begin{array}{l}65.7^{\mathrm{b}} \\
\pm 5.1\end{array}$ & $\begin{array}{l}58.4^{\mathrm{b}} \\
\pm 1.2\end{array}$ & $\begin{array}{l}61.4^{\mathrm{a}} \pm \\
0.1\end{array}$ & $\begin{array}{l}=65.3^{\mathrm{a}} \\
\pm 4.9\end{array}$ & $\begin{array}{l}56.9^{\mathrm{b}} \pm \\
5.9\end{array}$ \\
\hline $\mathrm{AI}(\%)$ & $\begin{array}{l}76.3^{\mathrm{a}} \\
\pm 1.5\end{array}$ & $\begin{array}{l}78.7^{\mathrm{a}} \pm \\
2.0\end{array}$ & $\begin{array}{l}83.6^{\mathrm{a}} \\
\pm 3.9\end{array}$ & $\begin{array}{l}77.6^{\mathrm{a}} \\
\pm 3.7\end{array}$ & & & & & & & & & $\begin{array}{l}64.9^{\mathrm{b}} \\
\pm 1.7\end{array}$ & $\begin{array}{l}68.3^{\mathrm{ab}} \\
\pm 2.2\end{array}$ & $\begin{array}{l}74.7^{\mathrm{a}} \\
\pm 3.4\end{array}$ & $\begin{array}{l}67.1^{\mathrm{ab}} \\
\pm 3.3\end{array}$ \\
\hline $\begin{array}{l}\text { MDA }\left(\mu \mathrm{M} / 10^{9}\right. \\
\text { sperms })\end{array}$ & & & & & & & & & & & & & $\begin{array}{l}46.6^{\mathrm{a}} \pm \\
21.9\end{array}$ & $\begin{array}{l}=33.6^{\mathrm{a}} \pm \\
9.3\end{array}$ & $\begin{array}{l}35.2^{\mathrm{a}} \\
12.1\end{array}$ & $\begin{aligned}= & 34.6^{\mathrm{a}} \pm \\
& 11.2\end{aligned}$ \\
\hline
\end{tabular}

Superscripts a, b indicates significance $(\mathrm{p}<0.05)$ differences among the treatments; Superscripts A, B, C, D indicate differences among the storage periods.

Table 2: Effect of supplementation of glutathione peroxidase (1.5-2.5 IU/ml) to TEY extender on sperm attributes during storage of Labrador dog semen at $4{ }^{\circ} \mathrm{C}$ for $72 \mathrm{hrs}$

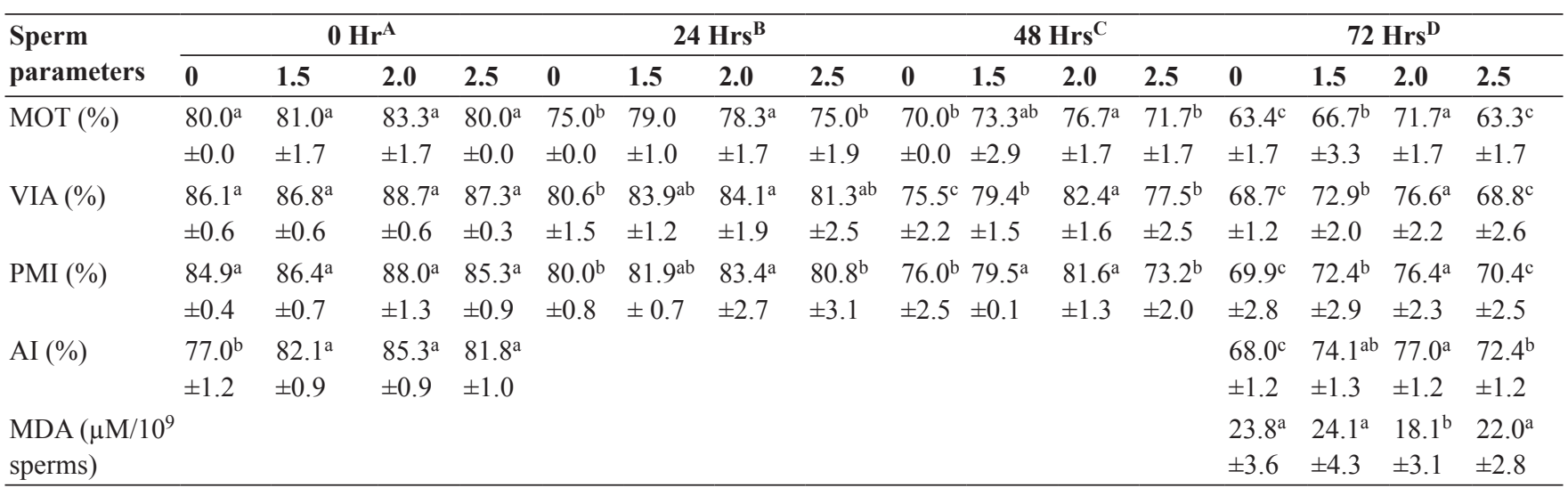

Superscripts $a, b$ indicate significance $(\mathrm{p}<0.05)$ differences among the treatments; Superscripts A, B, C, D indicate differences among the storage periods.

\section{Effect of Glutathione peroxidase on storage of semen at $4^{\circ} \mathrm{C}$}

The values for motility, viability and plasma membrane integrity were significantly $(\mathrm{p}<0.05)$ high in TEY extender supplemented with 1.5 and 2.0 IU GPX / ml compared to control and other doses at $24 \mathrm{hrs}$ of preservation (Table $2)$. However, these values remained significantly $(p<0.05)$ high in TEY extender supplemented with 2.0 IU GPX / $\mathrm{ml}$ at 48 and $72 \mathrm{hrs}$ of preservation. Acrosome integrity was also significantly $(\mathrm{p}<0.05)$ high in $2.0 \mathrm{IU}$ GPX / ml supplementation at $72 \mathrm{hrs}$ of preservation. Corresponding to sperm attributes, MDA concentration was significantly $(p<0.05)$ less in the presence of 2.0 IU GPX / $\mathrm{ml}$ compared to control and other doses.

\section{Effect of Catalase on storage of semen at $4^{\circ} \mathrm{C}$}

Supplementation of TEY extender with $200 \mu \mathrm{g} / \mathrm{ml}$ catalase resulted in significantly $(\mathrm{p}<0.05)$ higher motility, viability 
and plasma membrane integrity compared to control and other doses at 24, 48 and $72 \mathrm{hrs}$ of preservation (Table 3 ). Acrosome integrity also remained significantly $(p<0.05)$ higher in $200 \mu \mathrm{g} / \mathrm{ml}$ dose compared to control and other doses. Surprisingly, MDA concentration in the presence of $200 \mu \mathrm{g} / \mathrm{ml}$ catalase was non-significantly $(\mathrm{p}>0.05)$ higher than control and $100 \mu \mathrm{g} / \mathrm{ml}$ and lower than $300 \mu \mathrm{g} / \mathrm{ml}$.

\section{Effect of Glutathione on storage of semen at $4^{\circ} \mathrm{C}$}

Values for motility, viability and plasma membrane integrity were significantly $(\mathrm{p}<0.05)$ high in extender supplemented with $7.5 \mu \mathrm{M} / \mathrm{ml}$ GSH compared to control and other doses from $24-72 \mathrm{hrs}$ of preservation (Table $4)$. Acrosome integrity was significantly $(p<0.05)$ higher in $7.5 \mu \mathrm{M} / \mathrm{ml} \mathrm{GSH}$ compared to control. But difference between different doses of GSH was non-significant $(p>0.05)$. Similar to catalase supplementation, MDA concentration was non-significantly $(p<0.05)$ high in the presence of $7.5 \mu \mathrm{M} / \mathrm{ml}$ GSH compared to control and other doses.

A gradual decline in sperm attributes was noticed with increase in time of preservation irrespective of the antioxidant treatment. This observation supported previous studies on preservation of chilled dog spermatozoa in extenders (Iguer-ouada and Verstegen, 2001; Thiangtum et al., 2012). Similar to our observations, higher viability compared to motility was observed during preservation of semen at $5^{\circ} \mathrm{C}$ (Thiangtum et al., 2012). Moreover, plasma membrane integrity and acrosome integrity were also higher than motility at $72 \mathrm{hrs}$ of storage. Motility of Beagle dog spermatozoa was maintained up to 11 days by exchanging the old extender with fresh one (Verstegen et $a l ., 2005)$. It was also suggested that viable spermatozoa may be reactivated by medium supplementation in vitro or in vivo when interaction with the vagino-utero-oviductal secretion occurs. But semen was preserved in the same extender up to $72 \mathrm{hrs}$ of preservation in the present study. Therefore, decrease in sperm attributes with increasing storage period may be due to utilization of energy by the highly active spermatozoa and change in $\mathrm{pH}$ and osmolarity of extender. Reduction in motility with increasing storage period was attributed to the consumption of glucose, decrease in $\mathrm{pH}$ and metabolite accumulation (Rota et al., 1995; Verstegen et al., 2005). Many other factors may also influence the deterioration of chilled semen quality especially the lipid peroxidation due to oxidative stress during sperm storage in cooling condition.

It may be inferred that SOD, GPX, catalase and GSH acted in a dose dependent manner and 200 IU SOD, 2.0 IU GPX, $200 \mu \mathrm{g}$ catalase and $7.5 \mu \mathrm{M}$ GSH per ml semen were optimum doses for preservation of Labrador dog semen at $4^{\circ} \mathrm{C}$. Although motility, viability, plasma membrane integrity and acrosome integrity declined gradually in

Table 3: Effect of supplementation of Catalase $(\mu \mathrm{g} / \mathrm{ml})$ to TEY extender on sperm attributes during storage of Labrador dog semen at $4^{\circ} \mathrm{C}$ for $72 \mathrm{hrs}$.

\begin{tabular}{|c|c|c|c|c|c|c|c|c|c|c|c|c|c|c|c|c|}
\hline \multirow{2}{*}{$\begin{array}{l}\text { Sperm } \\
\text { Parameter }\end{array}$} & \multicolumn{4}{|c|}{$\mathbf{0} \mathbf{H r}^{\mathbf{A}}$} & \multicolumn{4}{|c|}{$24 \mathrm{Hrs}^{\mathrm{B}}$} & \multicolumn{4}{|c|}{$48 \mathrm{Hrs}^{\mathrm{C}}$} & \multicolumn{4}{|c|}{$72 \mathrm{Hrs}^{\mathrm{D}}$} \\
\hline & 0 & 100 & 200 & 300 & $\mathbf{0}$ & 100 & 200 & 300 & 0 & 100 & 200 & 300 & 0 & 100 & 200 & 300 \\
\hline MOT (\%) & $\begin{array}{l}75.0^{\mathrm{b}} \pm \\
0.0\end{array}$ & $\begin{aligned} & 75.0^{\mathrm{b}} \\
& \pm 5.8\end{aligned}$ & $\begin{array}{l}83.3^{\mathrm{a}} \\
\pm 3.3\end{array}$ & $\begin{array}{l}70.0^{\mathrm{b}} \pm \\
2.9\end{array}$ & $\begin{array}{l}=70.0^{\mathrm{b}} \pm \\
0.0\end{array}$ & $\begin{array}{l}=70.0^{\mathrm{b}} \pm \\
5.8\end{array}$ & $\begin{array}{l}78.3^{\mathrm{a}} \\
\pm 3.3\end{array}$ & $\begin{array}{l}65.7^{\mathrm{c}} \pm \\
2.3\end{array}$ & $\begin{array}{l}=61.7^{\mathrm{b}} \pm \\
0.7\end{array}$ & $\begin{array}{r} \pm 66.7^{\mathrm{b}} \\
\pm 3.3\end{array}$ & $\begin{array}{l}70.0^{\mathrm{a}} \\
\pm 2.9\end{array}$ & $\begin{array}{l}57.3^{\mathrm{c}} \\
\pm 3.7\end{array}$ & $\begin{array}{l}56^{7 b} \pm \\
1.7\end{array}$ & $\begin{array}{l}56.6^{\mathrm{b}} \pm \\
8.3\end{array}$ & $\begin{array}{l}65.7^{\mathrm{a}} \\
\pm 3.5\end{array}$ & $\begin{array}{l}43.3^{\mathrm{c}} \pm \\
7.3^{2}\end{array}$ \\
\hline VIA (\%) & $\begin{array}{l}83.9^{\mathrm{b}} \pm \\
2.2\end{array}$ & $\begin{aligned} & 81.2^{\mathrm{b}} \\
& \pm 2.9\end{aligned}$ & $\begin{array}{l}88.3^{\mathrm{a}} \\
\pm 4.6\end{array}$ & $\begin{array}{l}78.5^{\mathrm{c}} \pm \\
1.5\end{array}$ & $\begin{array}{l}=74.1^{\mathrm{b}} \pm \\
2.3^{2}\end{array}$ & $\begin{array}{l} \pm 72.5^{\mathrm{b}} \pm \\
2.5^{2}\end{array}$ & $\begin{array}{l}79.6^{\mathrm{a}} \\
\pm 4.1\end{array}$ & $\begin{array}{l}70.4^{\mathrm{b}} \pm \\
0.9\end{array}$ & $\begin{array}{l}+68.8^{\mathrm{b}} \pm \\
2.2\end{array}$ & $\begin{array}{c} \pm 70.0^{\mathrm{ab}} \\
\pm 3.3\end{array}$ & $\begin{array}{l}75.1^{\mathrm{a}} \\
\pm 3.0\end{array}$ & $\begin{array}{l}64.9^{\mathrm{c}} \\
\pm 1.2\end{array}$ & $\begin{array}{l}64.0^{\mathrm{b}} \pm \\
3.9\end{array}$ & $\begin{array}{l}=64.0^{\mathrm{b}} \pm \\
3.9\end{array}$ & $\begin{array}{l}70.3^{\mathrm{a}} \\
\pm 3.2\end{array}$ & $\begin{array}{l}55.1^{\mathrm{c}} \pm \\
1.9^{-}\end{array}$ \\
\hline PMI (\%) & $\begin{array}{l}85.1^{\mathrm{b}} \pm \\
2.1\end{array}$ & $\begin{array}{c}=80.0 \mathrm{~b}^{\mathrm{b}} \\
\pm 3.0\end{array}$ & $\begin{array}{l}89.7^{\mathrm{a}} \\
\pm 0.6\end{array}$ & $\begin{array}{l}80.4^{\mathrm{b}} \pm \\
1.2\end{array}$ & $\begin{array}{l}=75.0^{\mathrm{b}} \pm \\
1.2\end{array}$ & $\begin{array}{l} \pm 70.5^{\mathrm{b}} \pm \\
3.7\end{array}$ & $\begin{array}{l}81.6^{\mathrm{a}} \\
\pm 1.1\end{array}$ & $\begin{array}{l}70.2^{\mathrm{b}} \pm \\
1.4\end{array}$ & $\begin{array}{l}+70.6^{\mathrm{b}} \pm \\
2.9\end{array}$ & $\begin{array}{r} \pm 69.0^{\mathrm{b}} \\
\pm 7.1\end{array}$ & $\begin{array}{l}76.5^{\mathrm{a}} \\
\pm 3.7\end{array}$ & $\begin{array}{l}58.3^{\mathrm{c}} \\
\pm 5.1\end{array}$ & $\begin{array}{l}64.9^{b} \pm \\
4.4\end{array}$ & $\begin{aligned} & 61.7^{\mathrm{b}} \pm \\
& 10.7\end{aligned}$ & $\begin{array}{l}74.1^{\mathrm{a}} \\
\pm 3.0\end{array}$ & $\begin{array}{l}47.7^{\mathrm{d}} \pm \\
8.9^{ \pm}\end{array}$ \\
\hline $\mathrm{AI}(\%)$ & $\begin{array}{l}69.5^{\mathrm{a}} \pm \\
6.6\end{array}$ & $\begin{array}{l} \pm 74.9^{\mathrm{a}} \\
\pm 11.4\end{array}$ & $\begin{array}{l}84.8^{\mathrm{a}} \\
\pm 6.8\end{array}$ & $\begin{array}{l}68.7^{\mathrm{a}} \pm \\
2.8\end{array}$ & $t-$ & - & - & - & - & - & - & - & $\begin{array}{l}59.8^{\mathrm{a}} \pm \\
6.6\end{array}$ & $\begin{array}{l}65.4^{\mathrm{a}} \pm \\
12\end{array}$ & $\begin{array}{l}73.9^{\mathrm{a}} \\
\pm 12.0\end{array}$ & $\begin{array}{l}58.7^{\mathrm{a}} \pm \\
2.4\end{array}$ \\
\hline $\begin{array}{l}\mathrm{MDA}(\mu \mathrm{M} / 10 \\
\text { sperms })\end{array}$ & & - & - & - & - & - & - & - & - & - & - & - & $\begin{array}{l}35.4^{\mathrm{a}} \pm \\
10.1\end{array}$ & $\begin{array}{l}46.2^{\mathrm{a}} \pm \\
12.7\end{array}$ & $\begin{array}{l}66.6^{\mathrm{a}} \\
\pm 25.6 \\
\end{array}$ & $\begin{array}{l}77.4^{\mathrm{a}} \pm \\
31.6\end{array}$ \\
\hline
\end{tabular}

Superscripts a, b indicate significance $(\mathrm{p}<0.05)$ differences among the treatments; Superscripts A, B, C, D indicate differences among the storage periods. 
Table 4: Effect of supplementation of Glutathione $(\mathrm{mM} / \mathrm{ml})$ to TEY extender on sperm attributes during storage of Labrador dog semen at $4^{\circ} \mathrm{C}$ for $72 \mathrm{hrs}$

\begin{tabular}{|c|c|c|c|c|c|c|c|c|c|c|c|c|c|c|c|c|}
\hline \multirow{2}{*}{$\begin{array}{l}\text { Sperm } \\
\text { parameters }\end{array}$} & \multicolumn{4}{|l|}{ 0 $\mathrm{Hrs}^{\mathrm{A}}$} & \multicolumn{4}{|c|}{$24 \mathrm{Hrs}^{\mathrm{B}}$} & \multicolumn{4}{|c|}{48 Hrs $^{\mathrm{C}}$} & \multicolumn{4}{|c|}{$72 \mathrm{Hrs}^{\mathrm{D}}$} \\
\hline & 0 & 5.0 & 7.5 & 10.0 & $\mathbf{0}$ & 5.0 & 7.5 & 10.0 & $\mathbf{0}$ & 5.0 & 7.5 & 10.0 & $\mathbf{0}$ & 5.0 & 7.5 & 10.0 \\
\hline \multirow[t]{2}{*}{ MOT (\%) } & $73.3^{\mathrm{ab}}$ & $76.7^{\mathrm{ab}_{ \pm}}$ & $80.0^{\mathrm{a}}$ & $71.7^{b} \pm$ & $65.0^{\mathrm{b}} \pm$ & $\pm 60.0^{b}$ & $73.3^{\mathrm{a}}$ & $60.0^{\mathrm{b}} \pm$ & $\pm 56.7^{b}=$ & $\pm 56.7^{\mathrm{b}}$ & b $65.0^{\mathrm{a}}$ & $56.7^{b}$ & $48.3^{\mathrm{b}}$ & $50.0^{\mathrm{b}} \pm$ & $\pm 60.3^{\mathrm{a}}$ & $46.7^{b}$ \\
\hline & \pm 4.4 & 1.7 & \pm 0.0 & 3.3 & 0.0 & \pm 2.9 & \pm 1.7 & 2.9 & 1.7 & \pm 1.7 & \pm 0.0 & \pm 6.0 & \pm 1.7 & 0.0 & \pm 1.7 & \pm 5.7 \\
\hline \multirow[t]{2}{*}{ VIA (\%) } & $80.0^{\mathrm{ab}}$ & $81.2^{\mathrm{ab}}$ & $84.6^{\mathrm{a}}$ & $78.5^{b} \pm$ & $70.4^{\mathrm{ab}}$ & $65.9^{\mathrm{b}}$ & $78.3^{\mathrm{a}}$ & $67.8^{\mathrm{ab}}$ & $61.9^{\mathrm{ab}}$ & $60.4^{\mathrm{b}}$ & b $70.8^{a}$ & $62.5^{\mathrm{ab}}$ & $53.8^{\mathrm{b}}$ & $54.7^{\mathrm{b}} \pm$ & $\pm 62.8^{\mathrm{a}}$ & $53.5^{\mathrm{b}}$ \\
\hline & \pm 3.9 & \pm 1.9 & \pm 0.9 & 5.1 & \pm 2.9 & \pm 1.7 & \pm 0.9 & \pm 6.2 & \pm 0.4 & \pm 2.4 & \pm 1.0 & \pm 7.2 & \pm 1.2 & 3.1 & \pm 2.2 & \pm 4.5 \\
\hline \multirow[t]{2}{*}{ PMI (\%) } & $67.6^{\mathrm{b}} \pm$ & $65.6^{\mathrm{b}} \pm$ & $76.0^{\mathrm{a}}$ & $52.4^{\mathrm{b}_{ \pm}}$ & $63.3^{\mathrm{b}} \pm$ & $\pm 59.9^{b}$ & $70.8^{\mathrm{a}}$ & $59.2^{\mathrm{b}} \pm$ & $\pm 55.3^{\mathrm{b}}=$ & $\pm 52.7^{\mathrm{b}}$ & ${ }^{b} 63.7^{a}$ & $55.3^{\mathrm{b}}$ & $45.9^{\mathrm{b}}$ & $47.8^{\mathrm{b}} \pm$ & $=61.4^{\mathrm{a}}$ & $49.0^{\mathrm{b}}$ \\
\hline & 1.0 & 3.2 & \pm 3.0 & 3.8 & 1.9 & \pm 3.1 & \pm 1.6 & 7.9 & 2.7 & \pm 2.0 & \pm 1.1 & \pm 2.2 & \pm 3.3 & 2.8 & \pm 7.7 & \pm 6.0 \\
\hline \multirow[t]{2}{*}{$\mathrm{AI}(\%)$} & $70.0^{\mathrm{b}_{ \pm}}$ & $69.2 b \pm$ & $79.5^{\mathrm{a}}$ & $74.7^{\mathrm{b}} \pm$ & & & & & & & & & $57.5^{\mathrm{b}}$ & $61.3^{\mathrm{ab}}$ & $68.9^{\mathrm{a}}$ & $59.2^{\mathrm{ab}}$ \\
\hline & 4.7 & 1.4 & \pm 4.1 & 4.9 & & & & & & & & & \pm 5.0 & \pm 2.3 & \pm 5.1 & \pm 3.3 \\
\hline \multirow{2}{*}{$\begin{array}{l}\text { MDA }\left(\mu \mathrm{M} / 10^{9}\right. \\
\text { sperms })\end{array}$} & & & & & & & & & & & & & $52.7^{\mathrm{a}}$ & $55.9^{\mathrm{a}}$ & $63.5^{\mathrm{a}}$ & $50.5^{\mathrm{a}}$ \\
\hline & & & & & & & & & & & & & \pm 26.7 & \pm 29.3 & \pm 23.4 & \pm 33.6 \\
\hline
\end{tabular}

Superscripts a, b indicate significance $(\mathrm{p}<0.05)$ differences among the treatments; Superscripts A, B, C, D indicate differences among the storage peri.

the presence of optimum dose of antioxidants, but still remained $>60 \%$ at $72 \mathrm{hrs}$ of preservation. Contrary to our observations, extender containing 100, 400 and 1,600 U/ $\mathrm{ml}$ of CAT or SOD or the combination did not enhance sperm motility, viability and acrosomal integrity during storage at $5^{\circ} \mathrm{C}$ (Thiangtum et al., 2012). Comparable to our observations in dog, supplementing CAT and SOD and their combination to extender resulted in positive effect on chilled semen and during storage at $5^{\circ} \mathrm{C}$ in ram (Maxwell and Stojanov, 1996). Addition of CAT did not affect the maintenance of sperm motility during storage at $5^{\circ} \mathrm{C}$ in equine (Aurich et al., 1997; Ball et al., 2001). The addition of CAT to Tris-Lecithin extender did not improve even chilled dog semen (Beccaglia et al., 2009), but moderate benefit was reported when catalase at 100 $\mathrm{U} / \mathrm{ml}$ was added to Tris-glucose egg yolk (Michael et al., 2009). The addition of CAT (100 and $200 \mathrm{U} / \mathrm{mL}$ ) reduced the deleterious effects of cooling on total motility in ram sperm maintained at $5^{\circ} \mathrm{C}$ for $24 \mathrm{~h}$, although it did not affect the functionality of the sperm membranes (Câmara et al., 2011).

Addition of GSH at a concentration of $7.5 \mu \mathrm{M} / \mathrm{ml}$ was an optimum dose in improving sperm attributes compared to control during storage of Labrador dog semen at $4^{\circ} \mathrm{C}$. Contrary to this observation, supplementation of the extender with $5 \mathrm{mM}$ GSH improved the chilled and frozen-thaw semen quality (Angrimani et al., 2018; Monteiro et al., 2009). (Strezezek et al., 2009) reported that the physiologically normal GSH concentration in canine semen was closer to $5 \mathrm{mM}$, which is recommended for supplementation of semen extender/freezing solution. Variation in optimum dose of GSH had been reported in other species, as GSH@2.5 mM, $1 \mathrm{mM}$ and $0.5 \mathrm{mM}$ could preserve sperm quality of equine (de Oliveira et al., 2013), boar (Zhang et al., 2013) and bull (Tuncer et al., 2010) during storage of semen at $4^{\circ} \mathrm{C}$, respectively.

Among the three concentrations of GPX tested, only 2 $\mathrm{IU} / \mathrm{ml}$ supplementation to the extender could improve the sperm attributes in Labrador dogs. But, Del Prete et al. (2018) supplemented 15 IU of GPX and observed an improvement in DNA integrity during storage of semen at $4^{\circ} \mathrm{C}$ in fertile and hypo-fertile dogs.

It may be inferred from these studies that the different species respond variably to the antioxidants. Moreover, influence of environment including temperature, extenders and endogenous antioxidants may also be possible reasons behind variation. Different concentration of antioxidants should be evaluated for a species under investigation as per the conditions of particular lab, as optimum dose for one species may not be effective or may be toxic for the other species due to the change in physiological condition of semen extender. We also observed higher doses of antioxidants as toxic to dog spermatozoa. Addition of $400 \mathrm{mM}$ and $10 \mathrm{mM}$ GSH caused negative effects on ram (Câmara et al., 2011) and canine sperm (Ogata et al., 2015). In ram, survival of spermatozoa will increase when 
the dosage of SOD and CAT added to extender increases. However, CAT at dosage higher than $200 \mathrm{U} / \mathrm{ml}$ was toxic to ram spermatozoa (Maxwell and Stojanov, 1996).

There was no significant $(\mathrm{p}<0.05)$ difference in MDA concentration in control and antioxidant treated samples except GPX. Rather MDA concentration was nonsignificantly high in the presence of optimum catalase and GSH concentration. The addition of catalase in semen extenders resulted in significantly $(\mathrm{p}<0.0005)$ lower total ROS values compared with the controls in canine (Michael et al., 2009). GSH supplementation at a concentration of $5 \mathrm{mM}$ and $1 \mathrm{mM}$ inhibited lipid peroxidation levels in postthaw canine spermatozoa (Ogata et al., 2015) and beetal buck semen storage at $4^{\circ} \mathrm{C}$ (Sarangi et al., 2017). But, similar to our observations, MDA content of GSH treated groups was higher than that of the control group during storage at $4^{\circ} \mathrm{C}$ (Zhang et al., 2016). In spite of higher MDA content viz a viz lipid peroxidation in semen samples supplemented with optimum antioxidants concentration, the sperm attributes were higher compared to control and other doses.

In the dog, seminal fluid has been found to be deleterious for the in vitro preservation of sperm quality (Rota et al., 1995). It is also indicated that its presence before semen cryopreservation is also detrimental to sperm quality after thawing (Sirivaidyapong et al., 2001; Niżański, 2006). Therefore, seminal plasma was removed for processing, extension and preservation of semen at $4{ }^{\circ} \mathrm{C}$ and main disadvantage of this method is the elimination of natural antioxidants from seminal plasma (Strzeżek et al., 2009). Semen has enzymatic (SOD, catalase and GPx) and nonenzymatic antioxidants (Michael et al., 2009). Canine spermatozoa contain antioxidant enzymes, such as SOD and GPX for protection against the cytotoxic effects of internally generated ROS (Strzeżek et al., 2009). However, the sparse cytoplasmic volume in the sperm cell midpiece limits its antioxidant capacity (Aitken et al., 1996). Moreover, intracellular antioxidant enzymes do not protect acrosomal and plasma membrane, so this must be protected by seminal plasma (Iwasaki and Gagnon 1992; Zini et al., 2002). ROS are produced by sperm in physiological conditions and play an important role on mammal sperm function (Rota et al., 1995; Beccaglia et al., 2009). ROS in low concentrations are necessary to achieve sperm fertilizing capacity, as they are involved in sperm kinetic, capacitation, hyper activation and fusion of the oocyte with the spermatozoa (Rota et al., 1995). Free radical concentration is controlled by the antioxidants present in seminal plasma and spermatozoa. The contribution of each antioxidant system varies among species and it is believed that there is a synergistic action between them (Michael et al., 2009). Antioxidants can act by inactivating free radicals (GSH, SOD, catalase, GPX and vitamin E) or repairing the oxidative stress lesions (Ferreira and Matsubara, 1997, ascorbate, glutathione reductase GR and GPX). In the absence of seminal plasma, free radical concentration was maintained by supplemented antioxidants. Therefore, higher concentration of MDA and higher values of sperm attributes in the presence of optimum dose of antioxidants compared to control reveals that accomplished level of oxidative stress in preserved semen was not disproportionate and toxic to the spermatozoa. Otherwise, excessive production of free radical is responsible for alterations in semen quality, caused by decrease in sperm motility, as well as protein and DNA damage, resulting in cell apoptosis (Griveau and Le Lannou, 1997; Lucio et al., 2016). It may be revealed that supplementation of optimum dose of antioxidants to the extender fulfilled the function of seminal plasma antioxidants and resulted in boosted sperm attributes in preserved semen.

\section{CONCLUSION}

It was concluded that 200 IU SOD, 2.0 IU catalase, $200 \mu \mathrm{g}$ GPX and $7.5 \mu \mathrm{M}$ GSH per $\mathrm{ml}$ were optimum concentrations to be supplemented to the extender for positive effect. Supplementation of enzymatic and nonenzymatic antioxidants could improve sperm attributes by maintaining proportionate level of oxidative stress during preservation of Labrador dog semen at $4^{\circ} \mathrm{C}$ for $72 \mathrm{hrs}$. Artificial insemination using liquid semen supplemented with antioxidants and exhibiting enhanced sperm attributes will definitely results in achieving higher fertility rate.

\section{ACKNOWLEDGEMENTS}

This work was funded by a Research Grant from Department of Biotechnology, Ministry of Science and Technology, New Delhi (SAN No.102/IFD/SAN/5331/2017-2018). 


\section{REFERENCES}

Agarwal, A., Saleh, R.A. and Bedaiwy, M.A. 2003. Role of reactive oxygen species in the pathophysiology of human reproduction. Fertil. Steril., 79: 829-843.

Aitken, R.J., Buckingham, D.W., Carreras, A. and Irvine. D.S. 1996. Superoxide dismutase in human sperm suspension: relationship with cellular composition, oxidative stress, and sperm function. Free Radic. Biol. Med., 21: 495-504.

Angrimani, S.R., Nichi, M., Brito, M.M., Kawai, G.K.V., Rui, B.R., Losano, J.D.A., Vieira, N.M.G., Francischini, M.C.P., Cruz, D.S.G., Queiroz-Hazarbassanov, N., Massoco, C.O. and Vannucchi, C.I. 2018. The use of reduced glutathione (GSH) as antioxidant for cryopreserved sperm in dogs. Arq. Bras. Med. Vet. Zootec., 70: 419-428.

Aurich, J.E., Schönherr, U., Hoppe, H. and Aurich, C. 1997. Effects of antioxidants on motility and membrane integrity of chilled-stored stallion semen. Theriogenology, 48: 185-192.

Ball, B.A., Medina, V., Gravance, C.G. and Baumber, J. 2001. Effect of antioxidants on preservation of motility, viability and acrosomal integrity of equine spermatozoa during storage at $5^{\circ} \mathrm{C}$. Theriogenology, 56: 577-589.

Beccaglia, M., Anastasi, P., Chigioni, S. and Luvoni, G.C. 2009. Tris-Lecithin Extender Supplemented with Antioxidant Catalase for Chilling of Canine Semen. Reprod. Domest. Ani., 44: 345-349.

Buege, J.A. and Aust, S.D. 1978. Microsomal lipid peroxidation. Methods Enzymol., 52: 302-310.

Câmara, D.R., Mello-Pinto, M.M., Pinto, L.C., Brasil, O.O., Nunes. J.F. and Guerra, M.M. 2011. Effects of reduced glutathione and catalase on the kinematics and membrane functionality of sperm during liquid storage of ram semen. Small Rumin. Res., 100: 44-49.

de Oliveira, R.A., Wolf, C.A., de Oliveira Viu, M.A. and Gambarini, M.L. 2013. Addition of glutathione to an extender for frozen equine, semen. J. Equine Vet. Sci., 33: 1148-1152.

Del Prete, C.F.C., Tafuri, S., Pasolini, M.P., Della Valle, G., Palumbo, V., Abbondante, L., Calamo, A., Barbato, V., Gualtieri, R., Talevi, R. and Cocchia, N. 2018. Effect of superoxide dismutase, catalase, and glutathione peroxidase supplementation in the extender on chilled semen of fertile and hypofertile dogs. J. Vet. Sci., 19: 667-675.

Ferreira, A.L.A. and Matsubara, L.S. 1997. Radicais livres. conceitos, doenças relacionadas, sistema de defesa e estresse oxidativo. Rev. da Assoc. Méd. Bras., 43: 61-68.

Griveau, J.F., Le Lannou, D. 1997. Reactive oxygen species and human spermatozoa: physiology and pathology. Int. J. Androl., 20: 61-69.

Iguer-ouada, M. and Verstegen, J.P. 2001. Long-term conservation of chilled canine semen: effect of commercial and laboratory-prepared extenders. Theriogenology, 55: 671684 .

Iwasaki, A. and Gagnon, C. 1992. Formation of reactive oxygen species in spermatozoa of infertile patients Fertil. Steril., 57: 409-416,

Jeyendran, R.S., Vander, V.H.H., Perez-Pelaez, M., Crabo, B.G. and Zaneveld, L.J.D. 1984. Development of an assay to assess the functional integrity of the human sperm membrane and its relationship to other semen characteristics. J. Reprod. Fertil., 70: 219-228.

Kasimanickam, V.R., Kasimanickam, R.K., Memon, M.A. and Rogers, H.A. 2012. Effect of extenders on sperm mitochondrial membrane, plasma membrane and sperm kinetics during liquid storage of canine semen at 5 degrees $\mathrm{C}$. Ani. Reprod. Sci., 136: 139-145.

Kawakami, E., Taimura, A., Sakuma, M. andTakano, M. 2007. Superoxide Dismutase and Catalase Activities in the Seminal Plasma of Normozoospermic and Asthenozoospermic Beagles. J. Vet. Med., 69: 133-136.

Linde-Forsberg, C. and Forsberg M. 1993. Results of 527 controlled artificial inseminations in dogs. J. Reprod. Fertil., (Supplement) 47: 313-323.

Lucio, C.F., Silva, L.C., Regazzi, F.M. et al. 2016. Effect of reduced glutathione (GSH) in canine sperm cryopreservation: In vitro and in vivo evaluation. Cryobiology, 72:135-140.

Maxwell, W.M.C. and Stojanov, T. 1996. Liquid storage of ram semen in the absence or presence of some antioxidants. Reprod. Fertil. Dev., 8: 1013-1020.

Michael, A.J., Alexopoulos, C., Pontiki, E.A., HadjipavlouLitina, D.J., Saratsis, P., Ververidis, H.N. and Boscos, C.M. 2009. Effect of antioxidant supplementation in semen extenders on semen quality and reactive oxygen species of chilled canine spermatozoa. Anim. Reprod. Sci., 112: 119135 .

Monteiro, J.C., Goncalves, J.S., Rodrigues, J.A. et al. 2009. Influence of ascorbic acid and glutathione antioxidants on frozenthawed canine semen. Reprod. Domest. Anim. Zuchthyg., 44: 359-362.

Niżański, W. 2006. Intravaginal insemination of bitches with fresh and frozen-thawed semen with addition of prostatic fluid: use of an infusion pipette and the Osiris catheter. Theriogenology, 66, 470-483.

Ogata, K., Sasaki, A. and Kato, Y. et al. 2015. Glutathione supplementation to semen extender. Reprod. Dev., 61: 116122.

Rota, A., Strom, B. and Linde-Forsberg, C. 1995. Effects of seminal plasma and three different extenders on canine semen stored at $4^{\circ} \mathrm{C}$. Theriogenology, 44: 885-900. 
Sarangi, A., Singh, P., Virmani, M., Yadav, A.S., Sahu, S., Ajithakumar, H.M., Kumari, A. and Rath, A.P. 2017. Effect of antioxidants supplementation on the quality of Beetal buck semen stored at $4^{\circ} \mathrm{C}$. Vet. World., 10: $1184-1188$

Sirivaidyapong, S., Ursem, P., Bevers, M.M. and Colenbrander, B. 2001. Effect of prostatic fluid on motility, viability and acrosome integrity of chilled and frozen-thawed dog spermatozoa. J. Reprod. Fertil., Suppl., 57: 383-386.

Strzeżek, R., Koziorowska-Gilun, M., Kowalówka, M. and Strzeżek, J. 2009. Characteristics of antioxidant system in dog semen. Pol. J. Vet. Sci., 12: 55-60.

Tapia, J.A., Macias-Garcia, B., Miro-Moran, A., OrtegaFerrusola, C., Salido, G.M., Peña, F.J. and Aparicio, I.M. 2012. The membrane of the mammalian spermatozoa: much more than an inert envelope. Reprod. Domest. Anim., 47: 6575 .

Thiangtum, K., Hori, T. and Kawakami, E. 2012. Effect of catalase and superoxide dismutase on motility, viability and acrosomal Integrity of Canine Spermatozoa during Storage at $5^{\circ} \mathrm{C}$. Thai J. Vet. Med., 42: 447-453.
Tuncer, P.B., Bucak, M.N., Büyükleblebici, S., Sarı̈zkan, S., Yeni, D. and Eken, A. et al. 2010. The effect of cysteine and glutathione on sperm and oxidative stress parameters of postthawed bull semen. Cryobiology, 61: 303-307.

Verstegen, J.P., Onclin, K. and Iguer-Ouada, M. 2005. Longterm motility and fertility conservation of chilled canine semen using egg yolk added Tris glucose extender: In vitro and in vivo studies. Theriogenology, 64: 720-733.

Yu, I., Songsasen, N., Godke, R.A. and Leibo, S.P. 2002. Differences among dogs in response of their spermatozoa to cryopreservation using various cooling and warming rates. Cryobiology, 44: 62-78.

Zhang, X.G., Qi, L. Wang, L.Q., Yang, G.S. and Hu, J.H. 2016. Effects of glutathione on sperm quality during liquid storage in boars. Anim. Sci. J., 87: 1195-1201.

Zini, A., Fischer, M.A., Mark, V., Phang, D. and Jarvi, K. 2002. Catalase-like and superoxide dismutase-like activities in human seminal plasma. Urol. Res., 30: 321-323. 
